\title{
Interazioni conversazionali, manipolazioni linguistiche e emergenza di abilità logiche in attività matematiche
}

\section{Conversational interactions, language manipulations and the emergence of logical abilities in mathematical activities}

\author{
Cristina Coppola ${ }^{\star}$, Antonio Iannaccone`, Monica Mollo e Tiziana Pacelli` \\ *Dipartimento di Matematica, Università degli Studi di Salerno - Italia \\ - Institut de psychologie et éducation, Université de Neuchâtel - Svizzera \\ 'Dipartimento di Scienze Filosofiche Umane e della Formazione, Università degli Studi di Salerno - Italia \\ -Dipartimento di Matematica e Applicazioni “R. Caccioppoli”, Università degli Studi di Napoli \\ "Federico II" - Italia
}

凶ccoppola@unisa.it, antonio.iannaccone@unine.ch, mmollo@unisa.it, tiziana.pacelli@unina.it

Sunto / In questo lavoro, in accordo con la prospettiva vygotskiana e focalizzando su alcuni aspetti della logica matematica, viene presentata una riflessione derivante da un insieme di ricerche che esplorano le relazioni tra linguaggio e sviluppo di abilità logiche. Si descrive la progettazione/implementazione di un dispositivo educativo, articolato in attività linguistico-manipolative realizzate in classi quarte di scuola primaria. Le attività si basano sulla costruzione $\mathrm{e}$ manipolazione di un linguaggio simbolico, socialmente condiviso, nato per far muovere un "bambino-robot" in una stanza. L'analisi qualitativa degli scambi sociali e del contenuto delle interviste di esplicitazione ha permesso di evidenziare come, attraverso il confronto dei diversi punti di vista, i bambini realizzano un processo dinamico di costruzione e negoziazione di significati matematici. In particolare, sono presentati alcuni temi comuni emersi dalle interviste che mostrano le modalità di elaborare le attività da parte dei bambini, collegate ad aspetti interindividuali e intraindividuali.

Parole chiave: linguaggio; abilità logiche; manipolazione di oggetti linguistici; sintassi e semantica; interazioni sociali.
Abstract / In this paper, according to the Vygotskian perspective and focusing on some aspects of mathematical logic, a reflection is presented based on some studies exploring the relationships between language and development of logical skills. We describe the design/implementation of an educational device, consisting of linguistic-manipulative activities carried out in 4 th grade school classes. The activities are based on the construction and manipulation of a symbolic language, socially shared, created to make a "child-robot" move in a room. The qualitative analysis of the social exchanges and of the content of the explicitation interviews allowed to highlight how, through the comparison among different points of view, children carry out a dynamic process of construction and negotiation of mathematical meanings. In particular, some main topics emerging from the interviews are presented, showing the ways in which children process activities, linked to inter-individual and intra-individual aspects.

Keywords: language; logical abilities; manipulation of linguistic objects; syntax and semantics; social interactions. 


\section{Introduzione}

In questo lavoro presenteremo una riflessione sul legame tra linguaggio e sviluppo di abilità logiche in situazioni di interazione sociale, che si basa su diversi studi effettuati nel corso degli anni, legati ad una indagine nata dall'incontro tra due gruppi di ricerca, uno in didattica della matematica e l'altro in psicologia dell'educazione.

La cornice teorica rimanda ai lavori classici del costruzionismo sociale genetico (Doise et al., 1975; lannaccone, 1984; Iannaccone \& Ligorio, 2001; Perret-Clermont, 1979), ai contributi della scuola storico-culturale di Vygotskij $(1990,1997)$ e ai numerosi studi in educazione matematica che evidenziano l'influenza del linguaggio e delle competenze linguistiche nei processi di insegnamento e apprendimento della matematica e nelle pratiche sociali della classe (ad esempio, Ferrari, 2002, 2021; Planas, 2018; Planas et al., 2018).

L'importanza del ruolo svolto dal linguaggio nei processi di insegnamento e apprendimento della matematica viene evidenziata, in Italia, anche nelle Indicazioni nazionali per il curricolo della scuola dell'infanzia e del primo ciclo d'istruzione (Ministero dell'istruzione, dell'università e della ricerca [MIUR], 2012). In esse si sottolineano diversi aspetti, come ad esempio: la centralità di attività in cui siano presenti la discussione e l'argomentazione; I'apprendimento visto come un processo, in cui sono coinvolte la negoziazione e la costruzione di significati, o cambiamenti di punti di vista; il linguaggio usato non solo per comunicare informazioni, ma anche come strumento per riflettere, operare, discutere e collaborare; la considerazione che alcune difficoltà di apprendimento della matematica siano dovute a difficoltà linguistiche (Ferrari, 2021).

In accordo con tali prospettive, nel corso degli anni sono state progettate e realizzate attività matematiche centrate sulla manipolazione linguistica e sull'interazione in classi di scuola primaria, focalizzando l'attenzione sullo studio di alcuni aspetti del linguaggio che sono tipici della logica matematica, come ad esempio gli aspetti sintattici e semantici.

Le attività hanno riguardato sia gli aspetti procedurali sia quelli assertivi del linguaggio, che giocano un ruolo fondamentale nello sviluppo del pensiero matematico dei bambini (ad esempio, Coppola et al., 2010, 2011a, 2011b, 2014, 2015, 2019). Gli aspetti procedurali sono, infatti, collegati ai processi di riconoscimento-produzione di un linguaggio (basti considerare, ad esempio, le grammatiche), all'abilità di "contare all'infinito", alle procedure di ricorsione e induzione che sottendono le operazioni aritmetiche (Gerla et al., 1990). Gli aspetti assertivi del linguaggio, invece, sono quelli relativi, ad esempio, allo studio delle proprietà dei numeri e dello spazio, alla proposta di assiomi, alla dimostrazione di un teorema. Gli studi su cui si riflette in questo articolo riguardano le attività basate sulla manipolazione di linguaggi procedurali.

In particolare, questo lavoro, si articola in due parti. Nella prima parte viene messo in evidenza lo scopo che accomuna le ricerche, in accordo con la prospettiva teorica - I'analisi della relazione tra linguaggio, visto come un oggetto da manipolare sul piano sociale e su cui riflettere, e l'emergenza di abilità logiche nei bambini. Nella seconda parte viene presentata la progettazione di quello che chiamiamo "dispositivo educativo", ovvero il design di un insieme di attività orientate a tale scopo, sulla base delle nostre riflessioni riguardanti i diversi studi fatti negli anni e gli effetti osservati nel corso delle sue implementazioni.

Dopo avere descritto la prospettiva teorica che guida la ricerca, illustreremo il dispositivo educativo progettato e implementato nel corso degli anni, e ci soffermeremo sull'analisi dei processi messi in atto da bambini di una classe coinvolti in alcune attività svolte nei primi anni della nostra ricerca. In particolare, discuteremo alcuni risultati, emersi dall'analisi effettuata sui diversi livelli possibili delle interazioni sociali e dialogiche (Doise, 1986; Doise et al., 1992), provenienti dall'osservazione delle attività e dall'analisi delle interviste a posteriori fatte ai bambini. L'intervista si ispira ad alcuni presupposti del colloquio di esplicitazione di Vermersch (1994/2005), metodologia che offre l'opportunità 
di raccogliere utili resoconti dell'esperienza vissuta. In campo educativo la verbalizzazione dell'esperienza vissuta costituisce un approccio conversazionale che sembra favorire negli allievi un'attività di riflessione del proprio operato e del proprio ruolo di attore.

\section{La cornice teorica}

\subsection{Linguaggio e attività cognitiva}

In psicologia si attribuisce una notevole importanza al linguaggio in quanto espressione di una dimensione simbolica, un dispositivo della mente per mediare i simboli, per comunicare e per interagire (Bruner, 2002; Vygotskij, 1990). Le ricerche in psicologia di matrice socio-costruttivista (Doise \& Mugny, 1981; Iannaccone, 2010; Iannaccone \& Perret-Clermont, 1993; Perret-Clermont, 1979) hanno posto in evidenza l'importanza delle pratiche relazionali, sociali e conversazionali nel processo educativo. La costruzione della conoscenza è un processo collettivo condiviso dai partecipanti, le capacità di ragionamento aumentano nell'interazione con i pari o con adulti più esperti. Nel suo modello, Vygotskij (1990) fa dipendere lo sviluppo cognitivo del bambino dalla interiorizzazione delle conoscenze che il bambino acquisisce nel corso delle interazioni sociali alle quali prende parte. In relazione con I'altro (adulto/bambino; bambino/bambino) e in funzione del contesto socioculturale di riferimento, vengono stabiliti sul piano sociale gli strumenti per comprendere, organizzare e sistematizzare la conoscenza. II dialogo rappresenta un'occasione importante di scaffolding, in quanto consente al bambino, insieme ai suoi partners, non solo di acquisire quel sistema di criteri per comprendere e interpretare l'esperienza, ma anche di sistematizzare le conoscenze già possedute, che sono però ancora disorganizzate e irrazionali: i concetti (o pseudoconcetti), posseduti dal bambino, vengono messi a confronto con altri livelli di categorizzazione della realtà esibiti dai partner e che possono assumere anche la forma di veri e propri concetti che rispettano i criteri di logicità del pensiero razionale.

L'emergenza nello spazio semantico collettivo di forme di concettualizzazione più evolute offrirà al bambino nuovi strumenti di concettualizzazione che saranno progressivamente interiorizzati. Si attiva così un processo di presa di coscienza e padronanza. L'interiorizzazione di concetti e di significati rappresenta per un bambino un atto di pensiero complesso che sostiene una serie di funzioni (come attenzione volontaria, memoria logica, I'astrazione, confronto ecc.) e al tempo stesso richiede livelli progressivamente più elevati di presa di coscienza. Appare chiaro come «i concetti scientifici non sono assimilati e non sono appresi dal bambino, non sono imparati a memoria, ma nascono e si formano per la grandissima tensione di tutta l'attività del suo pensiero» (Vygotskij, 1990, p. 215). Il linguaggio stesso è un elemento integrante di tale attività, è «parte di un'attività multimodale che coinvolge anche azioni e percezioni» (Arzarello \& Robutti, 2009, p. 245). D'altra parte «il pensiero può essere concettualizzato come un caso di comunicazione, cioè come comunicazione con sé stessi» ed esso «sorge come versione privata modificata della comunicazione interpersonale» (Sfard, 2001, p. 26). Veicolando l'interiorizzazione delle relazioni sociali e delle conoscenze costruite all'interno di tali relazioni, il linguaggio rappresenterà un artefatto culturale, sotto forma di una «ricostruzione interna di un'operazione esterna» (Vygotskij, 1978, p. 56) che descrive «il processo di costruzione della conoscenza individuale come generata da esperienze socialmente condivise» (Bartolini Bussi \& Mariotti, 2009, p. 277). La logica dell'azione precede la logica del pensiero, in quanto lo sviluppo della mente (passaggio dall'interpsichico all'intrapsichico) passa attraverso situazioni concrete, tangibili e strettamente connesse con l'esperienza e con il contesto socioculturale di riferimento (Coppola et al., 2019; Vygotskij, 1990). 


\subsection{Logica, linguaggio e insegnamento}

Nel nostro studio, non guardiamo alla logica matematica come strumento per i fondamenti della matematica - come è stata considerata storicamente - ma come espressione esplicita di alcuni aspetti riguardanti il linguaggio, il cui ruolo è stato enfatizzato con la nascita della moderna logica matematica, all'inizio del ventesimo secolo. L'attenzione al linguaggio matematico, alla distinzione tra linguaggio e metalinguaggio e alla nozione di interpretazione fa della logica uno strumento culturale di insegnamento a ogni livello scolastico (Ferrari \& Gerla, 2015). D'altra parte, il linguaggio, in tutte le sue forme, ha un ruolo fondamentale nei processi di apprendimento e nelle pratiche sociali delle classi, come è stato ampiamente riconosciuto (si veda ad esempio, Ferrari, 2004, 2021; Planas, 2018; Planas et al., 2018).

In particolare, focalizziamo l'attenzione sui seguenti aspetti: il linguaggio, che da strumento di comunicazione diventa anche oggetto di manipolazione e di riflessione; I'interpretazione del linguaggio, che può non essere univoca; gli oggetti matematici che nascono non solo tramite processi di astrazione dall'esperienza diretta, ma anche dall'oggettivazione del linguaggio, l'intuizione di una equivalenza tra diverse procedure e l'individuazione di regole di manipolazione di oggetti linguistici in accordo con questa equivalenza (Coppola et al., 2011b, 2014; Gerla, 1988).

Dal punto di vista didattico, la trasformazione del glinguaggio in un oggetto da manipolare può essere realizzata attraverso attività linguistico-manipolative, in cui gli oggetti matematici nascono dalla manipolazione del linguaggio e del metalinguaggio. La manipolazione di oggetti linguistici, "costruiti" dopo una negoziazione comunicativa, a partire da situazioni problematiche, può stimolare nei bambini coinvolti l'apprendimento dell'uso di segni come simboli. I simboli sono intesi come "strumenti" che supportano la realizzazione di azioni nel contesto in cui i bambini agiscono e aiutano a trovare strategie per risolvere problemi. «Da un lato, funzionano come strumenti che permettono agli individui di impegnarsi nella prassi cognitiva. Dall'altro, fanno parte di quei sistemi che trascendono I'individuo e attraverso i quali si oggettiva una realtà sociale» (Radford, 2000, p. 241).

Inoltre, l'idea che sia importante gettare le basi per un'alfabetizzazione simbolica già nella scuola primaria, è supportata dalla convinzione di diversi ricercatori che alcune delle difficoltà degli studenti nell'apprendimento della matematica siano legate alle difficoltà nell'acquisizione della rappresentazione simbolica dei contenuti e delle operazioni matematiche. In tal senso, I'acquisizione di una competenza simbolica, attraverso attività di manipolazione, potrebbe promuovere negli studenti una migliore abilità di astrazione. La nozione di astrazione in matematica può avere diverse interpretazioni: come generalizzazione, come decontestualizzazione, come reificazione. Molte ricerche sottolineano che queste tre componenti sono fondamentali e intervengono in modi diversi a seconda che ci si riferisca all'organizzazione di conoscenze già disponibili o allo sviluppo di idee matematiche (Ferrari, 2003). D'altra parte, anche la prospettiva bruneriana (Bruner, 1977, 1990), riguardante il passaggio dal pensiero esecutivo a quello simbolico e poi astratto sostiene che, per raggiungere l'astrazione, il bambino deve passare attraverso la manipolazione dell'oggetto reale, poi la manipolazione della sua rappresentazione.

La progettazione di un dispositivo educativo, incentrato su una serie di attività collaborative ad hoc, segue una precisa architettura, che favorisce il passaggio da una esperienza pratica e diretta - attraverso una serie di attività proposte dai ricercatori - ad una conoscenza riflessiva di concetti matematici (Coppola et al., 2019). Tale passaggio è reso possibile dalla creazione di uno spazio dialogico di confronto tra gli alunni, capace di attivare cambiamenti concettuali (Bakhtin, 1981; Vygotskij, 1990), che evolve continuamente in una dimensione temporale (nella dimensione della micro-storia del dialogo) e che diviene, a sua volta, uno spazio per il pensiero (Mollo, 2018; Perret-Clermont, 2005, 2015). Tali cambiamenti passano attraverso azioni concrete, confronto e dialogo tra alunni e ricercatori (Coppola et al., 2019). La creazione di un linguaggio condiviso, all'interno di uno spazio dialogico di confronto, e la sua manipolazione permettono di acquisire la consapevolezza che "il simbolo" presume un accordo nella comunità dei destinatari e che dipende in generale dal contesto d'uso. In 
questo senso, il pensiero, e, quindi, il pensiero matematico, non può essere considerato come una elaborazione individuale di informazioni, ma costituisce una vera e propria "forma di pratica sociale" (Radford et al., 2005; Wartofsky, 1979) da interiorizzare.

\section{La ricerca}

Lo scopo principale che accomuna le ricerche, in accordo con la prospettiva teorica delineata, è quello di analizzare la relazione tra linguaggio, visto come un oggetto da manipolare sul piano sociale e su cui riflettere, e l'emergenza di abilità logiche nei bambini. II design delle attività del dispositivo educativo (che sarà descritto più nel dettaglio nel paragrafo successivo) prevede la creazione e la manipolazione di semplici linguaggi simbolici, come risposta alla proposta di situazioni problematiche, in una continua articolazione tra sintassi e semantica.

Il lavoro dei bambini vede alternarsi momenti di lavoro in gruppo e momenti di discussione collettiva. Si è cercato di realizzare, come setting per le attività, una sorta di "luogo sicuro" che facilitasse il confronto tra punti di vista diversi, le interazioni sociali e la manipolazione di oggetti linguistici. Dal nostro punto di vista, e in accordo con le teorie socio-costruttiviste, la creazione di uno spazio (Bakhtin, 1981; Coppola et al., 2019; lannaccone \& Zittoun, 2014; Perret-Clermont, 2001, 2004) all'interno del quale i bambini si confrontano su ciò che a loro è sconosciuto/ignoto oppure su elementi che destabilizzano le loro opinioni, rappresenta per i partecipanti una fonte di riflessione, presa di coscienza di sé e delle proprie conoscenze. In quest'ottica, tale spazio va considerato all'interno di una dimensione dialogica delle interazioni (Bakhtin, 1981) e va inteso anche in termini di spazio per il pensiero (Perret-Clermont, 2001).

In questo caso, l'elaborazione della conoscenza da parte dei bambini avviene attraverso un processo induttivo (bottom-up) che parte dalla manipolazione "collettiva" del linguaggio. L'attività di manipolazione del linguaggio consiste nel comporre, scomporre, ricombinare e confrontare il linguaggio al fine di individuare, in quel determinato contesto, un linguaggio simbolico condiviso da tutti i bambini. L'attività, in linea con la prospettiva teorica, consiste nell'individuare regole condivise di manipolazione di oggetti linguistici.

La situazione problematica di partenza prevede quello che abbiamo chiamato "il task del bambino-robot": uno dei bambini della classe svolge il ruolo di un robot che si muove liberamente all'interno dell'aula. Gli altri bambini hanno il compito di trovare delle "istruzioni" per descrivere i movimenti del bambino-robot. A partire da questa situazione iniziale, attraverso successive consegne e discussioni, lo scopo è quello di far costruire ai bambini un semplice linguaggio simbolico, in cui ogni simbolo rappresenta un'istruzione per il robot e, dunque, ogni simbolo ha come significato un movimento elementare del robot. In questo linguaggio, una parola, ovvero una sequenza di simboli, rappresenta una sequenza di movimenti per il robot. Successivamente, i compiti proposti hanno come obiettivo quello di far manipolare il linguaggio creato, sulla base di regole ed interpretazioni legate al contesto per far riflettere su diverse nozioni matematiche, come ad esempio il concetto di equivalenza (Coppola et al., 2011b, 2014). Tutto ciò avviene sempre in un continuo "andirivieni" tra sintassi e semantica, che comporta anche una riflessione generale su questi concetti e sul fatto che uno stesso linguaggio possa avere interpretazioni diverse.

La domanda più generale da cui siamo partiti vuole indagare se attività riguardanti la manipolazione di "oggetti linguistici", ambientate in un contesto di interazione sociale e negoziazione di significati, all'interno di uno spazio dialogico di confronto (Bakhtin, 1981; Vygotskij, 1990), possano supportare nei bambini un cambiamento nella rappresentazione del linguaggio, da solo strumento di comunicazione ad oggetto di manipolazione. 
Più nello specifico delle attività e, dunque, riguardo a nozioni specifiche che ne sono state l'obiettivo didattico, le domande che hanno guidato i nostri studi sono state:

- In che modo, attraverso la creazione e la manipolazione "collettiva" dei simboli del linguaggio, i bambini possono appropriarsi della nozione di equivalenza?

- In che modo, opportunamente guidati e stimolati, i bambini possono riflettere sulla possibilità che uno stesso linguaggio possa avere interpretazioni diverse e, dunque, su alcuni aspetti della differenza tra sintassi e semantica?

\section{La metodologia: partecipanti, design delle attività, approccio ai dati}

\subsection{Partecipanti e metodologia di lavoro}

Nel corso di vari anni il nostro studio ha coinvolto classi quarte di diverse scuole primarie ${ }^{1}$ della provincia di Salerno, Italia, con la partecipazione alle attività progettate di bambini di età compresa tra gli 8 e i 10 anni. Le attività sono sempre state svolte durante le ore curriculari, con la presenza in classe di (tre) ricercatori e di un insegnante, in alcuni casi di matematica, in altri di italiano.

In accordo con la prospettiva vygotskiana, secondo cui le capacità di ragionamento dipendono in larga misura dall'interazione tra pari e con persone più esperte (Vygotskij, 1990), i bambini partecipavano alle attività in gruppi cooperativi. La cooperazione può essere l'origine di almeno tre trasformazioni del pensiero dell'individuo: in primo luogo, può essere utile per prendere coscienza di sé stessi; innalza la distinzione tra il soggettivo e l'oggettivo; infine, è fonte di regolazione. Queste trasformazioni permettono al bambino di superare l'egocentrismo (in senso piagetiano), portandolo a discernere altri punti di vista esistenti e diverse soluzioni dello stesso problema (Carugati \& Selleri, 2005). Inoltre, tale scelta metodologica è anche legata all'idea di progettare attività che stimolassero la comunicazione, in accordo con le ricerche che attribuiscono un ruolo importante ai processi comunicativi nello sviluppo del pensiero matematico (Sfard, 2001).

Più in particolare, I'organizzazione dello svolgimento delle attività si è ispirato ai cicli didattici (Bartolini Bussi \& Mariotti, 2009), con l'alternanza di varie fasi: esplorazione dell'artefatto, lavoro in gruppi con compiti mirati, discussione collettiva guidata da uno dei ricercatori. L'artefatto al centro delle attività non è da considerarsi un "artefatto materiale", ma un artefatto concettuale: il linguaggio costruito dai bambini che diventa oggetto da manipolare.

II ruolo dell'insegnante durante le attività è stato di coordinatore, mentre i ricercatori hanno svolto il ruolo di scaffolders (Wood et al., 1976), supportando i processi di pensiero durante la risoluzione dei compiti proposti, attraverso la proposta di domande e di spunti di riflessione, cercando di non intervenire troppo nella gestione delle attività e nel processo di risoluzione della situazione problematica.

\subsection{Design del dispositivo educativo}

II dispositivo educativo è stato organizzato in attività "goal-oriented", focalizzate sulla creazione e manipolazione di semplici linguaggi simbolici procedurali, con una continua articolazione tra sintassi e semantica. Sono previste diverse fasi, per ognuna delle quali sono forniti compiti che i bambini devono risolvere a partire da situazioni problematiche.

L'idea iniziale che ha ispirato il design del dispositivo educativo, da implementare nelle scuole primarie, è stata quella che caratterizza il Logo di Seymour Papert (1980), uno dei primi linguaggi di

1. La scuola primaria in Italia dura cinque anni e corrisponde alla scuola elementare nel Canton Ticino. 
programmazione orientato all'educazione dei bambini. L'idea del Logo era quella di far muovere una tartaruga-robot visibile sullo schermo del computer dando delle semplici istruzioni. Nel nostro caso, il tema ricorrente nelle attività del dispositivo educativo è il "bambino-robot": il ruolo del robot che deve muoversi realmente in una stanza viene affidato ad uno o più bambini.

Un'altra idea chiave a cui si è fatto riferimento nell'ideazione del dispositivo educativo è stata quella che guida il paradigma dell'informatica povera (Coppola et al., 2011a), secondo cui è possibile usare "materiale povero", come ad esempio cartoncini, al posto di un software e di un computer. L'obiettivo principale di questo paradigma è quello di supportare nei bambini lo sviluppo di alcune abilità, come, ad esempio, individuare i problemi, escogitare un linguaggio più o meno formale per codificarli, identificare le procedure adeguate a risolverli, descrivere queste procedure in modo univoco (Gerla, 1988; Gerla et al., 1990). In accordo con tale prospettiva, le istruzioni vengono date ed eseguite dai bambini che partecipano alle attività. II linguaggio, usato per le istruzioni, è costruito dai bambini stessi grazie a processi di interazione sociale, negoziazione e di argomentazione delle scelte. In questo modo i bambini possono svolgere il ruolo di creatori, manipolatori e interpreti di un programma basato su quel linguaggio.

Le attività del dispositivo educativo sono state progettate pensando a tre fasi principali: creazione del linguaggio, manipolazione del linguaggio, interpretazione del linguaggio.

\section{Fase 1: creazione del linguaggio}

Relativamente alla prima fase sono state progettate diverse tipologie di attività il cui scopo è la creazione di un semplice linguaggio procedurale, partendo da una data situazione e per risolvere un dato problema. Nel corso degli anni si è deciso di lasciare inalterate queste attività, in quanto, sulla base delle analisi effettuate, il loro design sembrava adatto per raggiungere lo scopo che ci eravamo prefissati. In questa fase i bambini vengono divisi in gruppi. In una prima attività viene scelto da loro un bambino che deve svolgere il ruolo di "bambino-robot". II compito di questo bambino è di muoversi liberamente nell'aula, facendo dei passi sul pavimento quadrettato (i pavimenti delle aule sono sempre ricoperti da mattonelle), e di arrivare ad una fissata posizione, partendo da un'altra posizione prestabilita. Il compito del resto della classe, divisa in gruppi, è descrivere su un foglio i movimenti del bambino-robot, facendo corrispondere una descrizione ad ogni movimento. Nel corso degli anni si è osservato che in questa prima attività i bambini tendono sempre ad usare inizialmente il linguaggio quotidiano per descrivere i vari movimenti del bambino-robot. Inoltre, le istruzioni create sono strettamente collegate al punto di vista "soggettivo" dei bambini che osservano e che scrivono. Ad esempio, tra le espressioni più usate si ritrovano gira a destra, gira a sinistra, in cui I'uso esplicito di destra e sinistra mostra la dipendenza dalla posizione dell'osservatore.

In una seconda attività viene proposta una nuova situazione problematica: vengono scelti due bambini-robot, che vengono posizionati inizialmente uno di fronte all'altro come in uno specchio. Il compito per ogni gruppo, ora, è di dare a turno una singola istruzione ai due robot con lo scopo di far raggiungere loro contemporaneamente una stessa posizione prestabilita. Anche relativamente allo svolgimento di questa attività, nel corso degli anni è stato osservato un comportamento simile: il compito stesso, per la sua stessa struttura, induce nei bambini un momento di crisi. ${ }^{2}$ Infatti, i bambini si rendono conto che, usando le istruzioni elaborate nell'attività precedente, non sono in grado di trovare una soluzione alla nuova situazione problematica loro proposta. Hanno bisogno di un linguaggio diverso che sia meno legato al contesto della situazione stessa, in cui in quel momento sono immersi. La discussione collettiva guidata dai ricercatori permette di negoziare la scelta delle istruzioni e di risolvere il compito proposto. Nelle nostre esperienze, i bambini sostituiscono un riferimento interno (ovvero legato al proprio punto di vista), come destra e sinistra, con uno esterno, come i punti cardi- 
nali. La scelta di usare i punti cardinali non è casuale, nel senso che generalmente vengono introdotti e studiati proprio in quegli anni scolastici. Inoltre, una prassi comune delle scuole in cui sono state svolte le attività, consisteva nell'etichettare le pareti delle aule con il punto cardinale corrispondente. In una conclusiva terza attività si attua il processo di istituzionalizzazione, atto sociale fondamentale affinché gli alunni, coinvolti precedentemente nelle situazioni problematiche in cui hanno costruito in interazione il linguaggio, riconoscano, guidati dal ricercatore, l'utilità e l'universalità del sapere costruito (D'Amore, 1999). «L'istituzionalizzazione della consegna è l'atto sociale attraverso il quale il maestro e l'allievo riconoscono la devoluzione» (Brousseau, citato da Perrin-Glorian, 1994, p. 128). In questa attività i simboli o le parole, creati dai vari gruppi, che descrivono i movimenti del robot, attraverso una discussione collettiva e continue negoziazioni, vengono trasformati in istruzioni di base per dare vita ad un unico linguaggio più sintetico, condiviso dall'intera classe. II linguaggio finale viene istituzionalizzato attraverso la scrittura su un cartellone dei simboli scelti. Dalle osservazioni effettuate in questi anni è emerso che in questa attività i bambini compiono un passaggio graduale dal linguaggio quotidiano ad uno "simbolico". Di seguito riportiamo le immagini di due cartelloni con due esempi di linguaggi creati in due diverse classi (Figura 1, Figura 2).

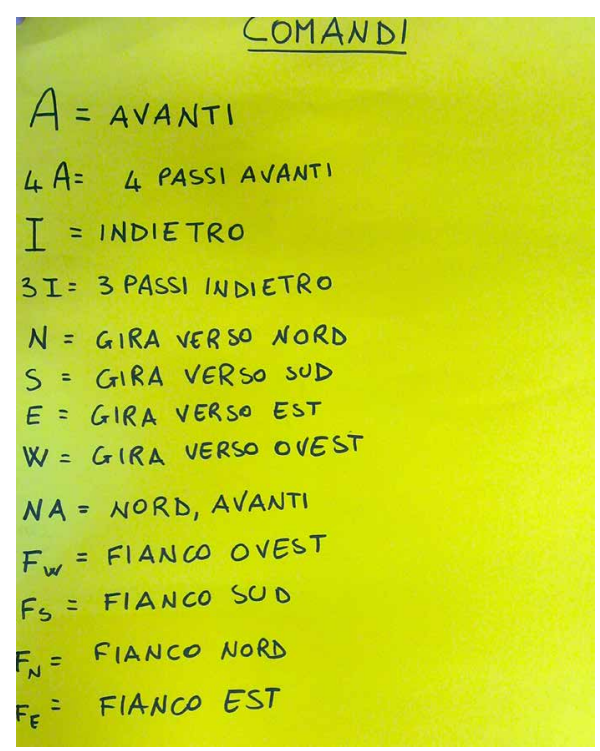

Figura 1. I simboli di un linguaggio creato e relativa interpretazione.

Con il simbolo $N$ i bambini intendevano l'istruzione per far girare il bambino-robot con lo sguardo rivolto verso Nord, rimanendo sulla stessa mattonella (analogamente per i simboli $S, E, W$ ). L'interpretazione attribuita al simbolo $F_{w}$ era quella legata al movimento del bambino-robot verso Ovest, senza girarsi, rimanendo cioè con lo sguardo nella stessa direzione in cui già si trovava (analogamente per i simboli $F_{E^{\prime}} F_{N}$ e $F_{S}$ ). Come possiamo osservare, in questo caso i bambini sul cartellone non scrivono solo le istruzioni elementari, ma anche alcune abbreviazioni, come ad esempio $3 /$ che abbrevia la parola ${ }^{3}$ III (utilizzando conoscenze pregresse) ed alcune parole brevi, come ad esempio NA, composta dall'accostamento dei due simboli $N$ ed $A$.

Da sottolineare che in attività di questo tipo, svolte in classi diverse, si sono ottenuti risultati simili

3. Con il termine parola intendiamo un "programma" costituito da una sequenza di simboli che possono essere interpretati come una successione temporale di istruzioni elementari. Ad esempio, AAIS è una parola costituita da quattro simboli che corrisponde ad una sequenza temporale di quattro istruzioni elementari e, quindi, di quattro azioni che il robot dovrebbe compiere: fai un passo avanti, fai un passo avanti, fai un passo indietro, gira verso sud. 
ma non identici. Questo dipende dal fatto che, essendo un'attività situata, legata al contesto in cui sono immersi i bambini, la scelta dei simboli - e la relativa interpretazione - è frutto di negoziazioni, di argomentazioni legate al contesto in cui si sta svolgendo l'attività, ai movimenti del robot e a ciò che i bambini, in quel contesto, hanno ritenuto importante per raggiungere lo scopo e per risolvere la situazione problematica. Ad esempio, i bambini di un'altra classe, coinvolta in un altro momento nell'implementazione delle attività, hanno prodotto il linguaggio che possiamo osservare nella Figura 2.

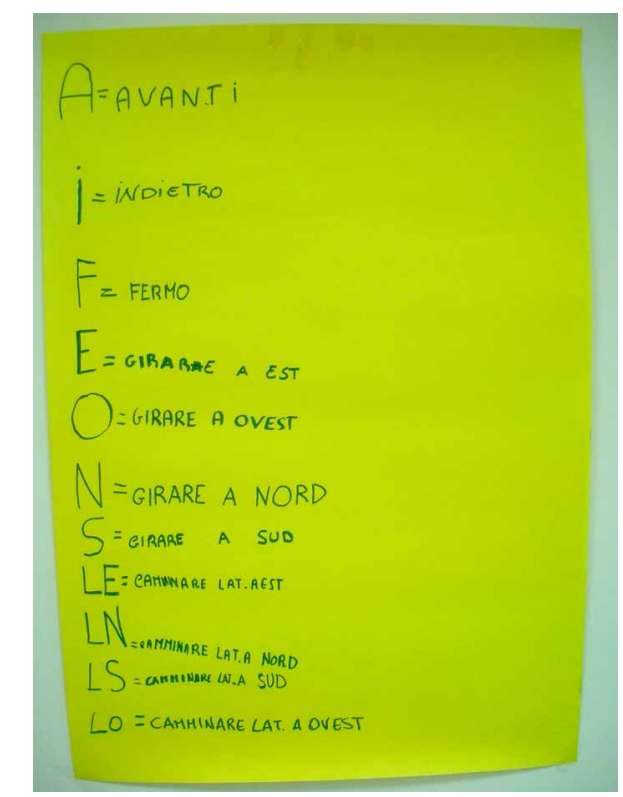

Figura 2. I simboli di un linguaggio creato e relativa interpretazione.

Con il simbolo $L_{N}$ i bambini intendevano descrivere un particolare movimento: il bambino-robot, senza cambiare la direzione verso cui è rivolto con lo sguardo, si sposta lateralmente di una mattonella verso il punto cardinale Nord. Analogo discorso vale per i simboli $L_{S^{\prime}} L_{E^{\prime}} L_{O^{\prime}}$.

In entrambe queste esperienze i bambini hanno scelto in modo spontaneo di usare i punti cardinali osservando l'ambiente in cui stavano svolgendo l'attività. In un contesto diverso, potrebbero scegliere un riferimento diverso.

\section{Fase 2: manipolazione del linguaggio}

Per la Fase 2 sono state progettate attività il cui scopo generale è quello di permettere ai bambini di manipolare il linguaggio costruito nella Fase 1 in un continuo andirivieni tra la sintassi del linguaggio e la sua semantica (interpretazione dei simboli e delle parole in riferimento al contesto). In particolare, per queste attività si è puntato sulla nozione di equivalenza. In accordo con alcuni studi, infatti, (ad esempio, Kieran, 1992; MacGregor \& Stacey, 1997) si ritiene fondamentale per uno studente riuscire a gestirla correttamente. Inoltre, la sua comprensione rappresenta un prerequisito fondamentale per l'apprendimento di nozioni matematiche più avanzate. Pertanto, lo scopo delle attività è quello di far riconoscere alcune regole di equivalenza del linguaggio costruito, per poi far sviluppare una maggiore abilità nella loro manipolazione. Bisogna precisare che, in questo contesto, due sequenze di simboli sono considerate equivalenti se rappresentano percorsi che, partendo da una stessa posizione iniziale, permettono al bambino-robot di arrivare in una stessa posizione finale. Una regola di equivalenza è, dunque, una regola sintattica, che permette di identificare due sequenze di simboli come equivalenti. Ad esempio, una regola di equivalenza può essere $A l=F$, poiché entrambe le sequenze di simboli producono lo stesso risultato per quanto riguarda la posizione finale, e considerando una stessa posizione iniziale. Di conseguenza, la parola $A I A A$ è equivalente alla parola $A A$. II termine 
"equivalente" è stato negoziato con i bambini, partendo dall'osservazione dell'effetto causato sui percorsi del robot dalle sequenze nei compiti dati. Anche in questa fase i bambini lavorano in gruppo ed ogni attività è caratterizzata da discussioni collettive finali, in cui, attraverso argomentazioni varie, i bambini, guidati dai ricercatori, negoziano il compito e le soluzioni ad esso.

Una prima attività progettata ed implementata riguarda la costruzione delle regole di equivalenza. Ogni gruppo riceve un foglio di carta (Figura 3) su cui è disegnata una griglia (che simula il pavimento dell'aula), sulla quale viene indicato un punto di partenza e viene scritta una sequenza di simboli del linguaggio costruito nella Fase 1. Sul foglio sono indicati anche i punti cardinali: $N, S, O, E$. In questo esempio ci si riferisce al linguaggio creato visibile nella Figura 2.

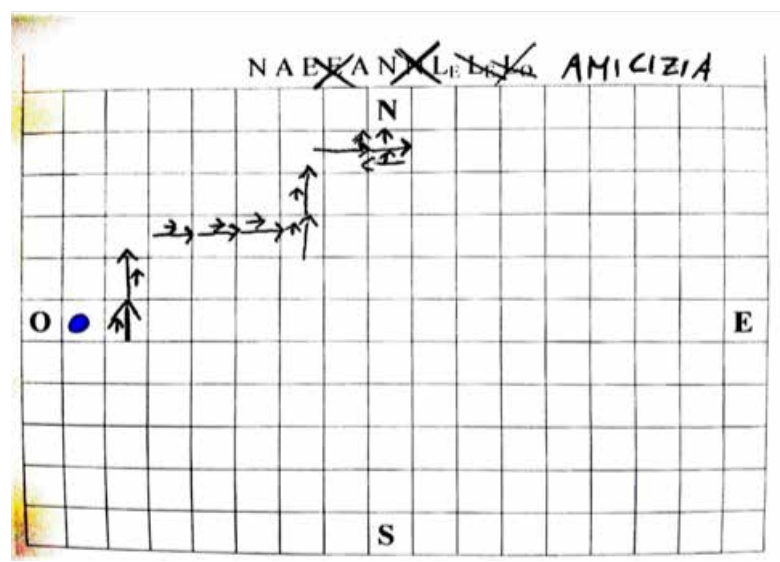

Figura 3. Esempio di rappresentazione su un foglio quadrettato dell'attività della Fase 2.

Il compito di ogni gruppo è rispondere alle seguenti domande:

- Disegna il percorso (corrispondente alla sequenza) e indica il punto di arrivo.

- Il percorso che hai disegnato per il robot è il più breve per raggiungere il punto d'arrivo?

- Puoi renderlo più breve eliminando alcune istruzioni?

- Cosa cancelli e come mai?

- Puoi sostituire alcune istruzioni con altre? Con quali? Come mai?

Un esempio di sequenza di simboli proposta ai bambini è la seguente:

$$
\text { NA A I A A E I I F F F A }
$$

Questa sequenza può essere resa più breve, per esempio, eliminando le sequenze di simboli $A /$ oppure $\left(F_{E} F_{W}\right)$ entrambe equivalenti a $F$, in quanto le interpretazioni di tutte è "nessun movimento". In tal modo si arriva alla sequenza

$$
\begin{gathered}
N A(A /) A A E A I\left(F_{E} F_{W}\right) A A A \\
N A A A E A / A A A .
\end{gathered}
$$

Nella Figura 3 possiamo osservare un altro esempio di sequenza di simboli proposta e come un gruppo di bambini (Gruppo Amicizia) I'ha rappresentata graficamente e ha effettuato le elisioni di alcuni simboli per renderla più breve.

Fase 3: interpretazione del linguaggio

Per questa fase sono state progettate attività il cui scopo è quello di far riflettere sulla differenza tra sintassi e semantica del linguaggio creato e, in particolare, sul fatto che uno stesso linguaggio possa avere interpretazioni diverse. A questo scopo sono state progettate e realizzate nel corso di più anni due attività diverse: la caccia al tesoro e il gioco dei mondi.

La caccia al tesoro

In un primo studio (Coppola et al., 2011a) è stata progettata e implementata un'attività molto simile 
a una caccia al tesoro. Durante questa attività i bambini sono divisi in due gruppi, che chiamiamo Gruppo A e Gruppo B. Inizialmente i due gruppi lavorano in aule diverse e hanno compiti diversi. Per il Gruppo A il compito è di nascondere il "tesoro" nell'aula e di creare una sequenza di simboli del linguaggio (di lunghezza limitata) che rappresenti un percorso che conduce al tesoro. La sequenza deve essere scritta su una griglia di carta. Si può suggerire che, poiché la sequenza creata sarà consegnata al Gruppo B, che la interpreterà per trovare il tesoro, il Gruppo A può trarre vantaggio dal creare una sequenza lunga con stringhe di simboli che possono essere semplificate. Per il Gruppo B, il compito è di manipolare ed eventualmente semplificare la sequenza di simboli ricevuti dal Gruppo A. La sequenza finale, ottenuta dopo la manipolazione, deve essere dettata da un membro del Gruppo $\mathrm{B}$ a un altro dello stesso gruppo, che svolgerà il ruolo di robot per trovare il tesoro. Prima della dettatura, il bambino-robot non conosce né la sequenza iniziale ricevuta dal suo gruppo né quella finale. Per raggiungere il tesoro, il bambino-robot deve eseguire il percorso solo sulla base delle informazioni che scrive sul suo foglio durante il dettato.

\section{Il gioco dei mondi}

In questa attività - descritta e analizzata nel dettaglio in (Coppola et al., 2015, 2019) - vengono proposte due o più interpretazioni diverse dei simboli di uno stesso linguaggio creato e manipolato nelle fasi precedenti. Vengono usati cartelloni di diversi colori per presentare diverse interpretazioni del linguaggio costruito (in Figura 4 un esempio relativo al linguaggio della Figura 2). In questa attività sono stati previsti sia momenti in cui i bambini-robot possono eseguire i comandi dei nuovi mondi, sia compiti da svolgere nei gruppi.

\begin{tabular}{|l|l|l|}
\hline Linguaggio & \multicolumn{1}{|c|}{$\begin{array}{c}\text { Interpretazione "mondo } \\
\text { arancione" }\end{array}$} & $\begin{array}{c}\text { Interpretazione "mondo } \\
\text { blu" }\end{array}$ \\
\hline$A$ & Avanti & Avanti \\
\hline$I$ & Indietro & Indietro \\
\hline$N$ & Gira a Sud & Alza la mano destra \\
\hline$S$ & Gira a Nord & Abbassa la mano destra \\
\hline$E$ & Gira a Ovest & Alza la mano sinistra \\
\hline$O$ & Gira a Est & Abbassa la mano sinistra \\
\hline$L_{N}$ & Cammina lateralmente a Sud & Gira a Nord \\
\hline$L_{S}$ & Cammina lateralmente a Nord & Gira a Sud \\
\hline$L_{E}$ & $\begin{array}{l}\text { Cammina lateralmente a } \\
\text { Ovest }\end{array}$ & Gira a Est \\
\hline$L_{O}$ & Cammina lateralmente a Est & Gira a Ovest \\
\hline$F$ & Fermo & Fermo \\
\hline
\end{tabular}

Figura 4. Esempi di interpretazioni diverse di uno stesso linguaggio.

Sono stati proposti più compiti con lo scopo di far costruire regole di equivalenza valide per i due nuovi mondi e di verificare se l'equivalenza è una proprietà che si conserva nel passaggio da un mondo a un altro, cioè se due sequenze di simboli, equivalenti in un mondo, continuano ad essere equivalenti anche in un altro mondo. In queste attività il focus risulta essere il continuo andirivieni tra sintassi e semantica dei simboli del linguaggio. Grazie alla loro struttura, i compiti (un esempio è presentato in Figura 5) permettono ai bambini di riflettere sul fatto che due sequenze sintatticamente identiche possono avere una semantica diversa a seconda del "mondo" in cui vengono usate e, di conseguenza, in un mondo possono valere delle regole di equivalenza e in un altro mondo altre regole. Nella progettazione sono state scelte interpretazioni opportune in modo che il mondo di partenza fosse isomorfo ad uno dei due mondi nuovi e non isomorfo all'altro. Per esempio, considerando come mondo di partenza (mondo giallo) quello con il linguaggio rappresentato in Figura 2, risulta che sia nel mondo di partenza che in quello arancione (Figura 4), la regola di equivalenza $L_{E} L_{O}=F$ vale, mentre questa regola non vale nel mondo blu (Figura 4). Pertanto, le parole $L_{E} L_{O}$ e $F$ sono equivalenti sia nel mondo giallo che in quello arancione, ma non sono equivalenti nel mondo blu. 
Leggete queste due sequenze di comandi:

NA I A $L_{E} L_{o}$ NFAF

1) Utilizzando le REGOLE del mondo GIALLO, le due sequenze sono equivalenti?

2) Utilizzando le REGOLE del mondo ARANCIONE, le due sequenze sono equivalenti?

Figura 5. Esempio di attività della Fase 3.

Nel prossimo paragrafo, dopo aver illustrato quali dati si sono ottenuti e come sono stati raccolti nel corso degli anni, ci soffermeremo sull'analisi e sui risultati riguardanti una delle prime progettazioni/ implementazioni del dispositivo educativo, ai quali si sono però aggiunti ulteriori risultati dovuti a studi successivi, di cui discuteremo solo nel paragrafo finale.

\subsection{Approccio ai dati}

Raccolta dati

Nel corso degli anni sono stati raccolti dati attraverso video-registrazioni, audio-registrazioni, ${ }^{4}$ successivamente trascritte, note osservative e tracce osservabili5 (Vermersch, 1994/2005).

Inoltre, in itinere e alla fine dell'implementazione del dispositivo educativo, i bambini sono stati sottoposti individualmente a una intervista narrativa, semi-strutturata con domande aperte, ispirata sia al costrutto dell'intervista di esplicitazione (Vermersch, 1994/2005) sia al resoconto biografico (Bruner, 1990). Per la loro stessa natura, non è mai stata fissata una durata prestabilita delle interviste. Tutte le interviste sono state audio-registrate e trascritte.

Sul piano metodologico appare essenziale un esplicito riferimento alla dimensione narrativa (Bruner, 2002) come modalità cognitiva attraverso cui i bambini organizzano e attribuiscono senso all'esperienza, interpretando la realtà al fine di rappresentare gli eventi e trasformarli in oggetto di riflessione (intrapsichico). Inoltre, l'intervista di esplicitazione ci è sembrata uno strumento adatto per raccogliere dati utili per il nostro studio, in quanto permette di verbalizzare l'azione: I'azione è intesa come esecuzione di un compito e, quindi, con l'espressione "verbalizzare l'azione" si intende l'operazione di trasformare in parole il processo messo in atto per svolgere un compito. Tale metodo, infatti, si basa su particolari tecniche di formulazione dei rilanci (domande, riformulazioni, silenzi) mirate a facilitare e assistere la verbalizzazione a posteriori di un particolare aspetto di una esperienza vissuta. In ogni attività che comporta l'esecuzione di compiti - come nel nostro caso - è fondamentale conoscere in dettaglio le modalità di esecuzione del compito stesso, perché la sola conoscenza del risultato finale del compito non è sufficiente per diagnosticare la natura e la causa di un fallimento o di un successo. Lo sviluppo dell'azione è l'unica fonte affidabile di inferenze. La sua conoscenza è necessaria per diversi aspetti: evidenziare i ragionamenti effettivamente svolti; identificare gli obiettivi effettivamente perseguiti (che spesso possono essere diversi da quelli che si suppone di perseguire); rintracciare le conoscenze teoriche che sono state effettivamente utilizzate nella pratica (che spesso possono essere diverse da quelle padroneggiate solo da un punto di vista teorico); evidenziare le rappresentazioni errate o i preconcetti che sono causa di problemi.

4. I bambini non hanno mai mostrato di essere a disagio per la presenza dei ricercatori e delle telecamere.

5. In accordo con Vermersch (1994/2005) le tracce sono i segni "concreti" prodotti dai bambini durante le attività, come note e compiti scritti nel corso e alla fine delle attività. 


\section{Analisi dei dati}

L'analisi dei dati, in questi anni, è stata di tipo qualitativo ed interpretativo ed è stata articolata in modo da considerare sia l'analisi del discorso e delle conversazioni dei bambini - avvenute durante lo svolgimento delle attività - che l'analisi delle loro narrazioni esplicitate attraverso le interviste.

In particolare, l'analisi del discorso e delle conversazioni riguarda gli scambi sociali (analisi di video e audio registrazioni), ovvero le modalità attraverso cui i bambini, in interazione, hanno co-costruito le soluzioni ai compiti loro proposti. Quest'analisi è stata portata avanti con vari strumenti teorici, ad esempio utilizzando la teoria dell'attività (Coppola et al., 2014), secondo cui tutti i processi psicologici si sviluppano nell'attività e l'azione, sempre mediata dagli strumenti culturali, rappresenta I'unica unità di analisi possibile (Leont'ev, 1975). L'analisi non mira tanto a comprendere il significato alla base dell'agire ma a studiare le attività orientate verso un obiettivo. Le attività si articolano in una serie di azioni, che sono intraprese consapevolmente per raggiungere uno scopo attraverso una serie di operazioni generate dall'azione che sono automatiche e indipendenti dalle caratteristiche dell'attività stessa. Pertanto, ogni attività è di per sé motivata da un oggetto che dà forma alla stessa attività, che si articola in azioni che generano operazioni all'interno di un determinato contesto sociale (Leont'ev, 1975; Ligorio \& Pontecorvo, 2010; Zucchermaglio, 1996).

L'analisi delle narrazioni, di cui un esempio è presentato in questo lavoro, concerne l'elaborazione cognitiva delle attività (passaggio vygotskiano dall'interpsichico all'intrapsichico) (Vygotskij, 1990), elaborata dai bambini, prima socialmente e poi individualmente, durante e alla fine delle attività. In particolare, è stata effettuata sulle trascrizioni delle interviste un'analisi del contenuto (Berelson, 1952; Blanchet, 1985), interpretando le modalità di risposta dei bambini. I ricercatori hanno prodotto separatamente le loro interpretazioni, assumendo il ruolo di giudici indipendenti. La procedura ha richiesto, come primo passo, l'individuazione nei resoconti delle interviste di unità lessicali che si riferiscono ad uno stesso tema o argomento. Queste sono state successivamente classificate in unità semantiche dando vita a dei temi. In caso di disaccordo per l'attribuzione delle unità lessicali ai temi, i giudici hanno discusso fino a raggiungere un pieno accordo. Nella costruzione dei temi è stato necessario rispettare alcune regole di classificazione: unicità del criterio di classificazione, esclusività reciproca dei temi, esaustività dell'insieme degli argomenti. Successivamente sono state valutate le frequenze delle unità lessicali in tutti i temi per poter prendere in considerazione i temi principali, cioè i temi con la frequenza più alta di unità lessicali.

\section{I risultati di una prima implementazione del dispositivo educativo}

Dopo la panoramica dei paragrafi precedenti sulla struttura delle ricerche e su come sono state articolate, ci soffermiamo sulla presentazione di una delle prime esperienze legate all'implementazione del dispositivo educativo e sull'analisi effettuata (Coppola et al., 2011a).

Nell'implementazione di cui parliamo è stata coinvolta una classe di quarta primaria dell'Istituto Comprensivo di Fisciano, Salerno. Hanno partecipato alle attività proposte 19 bambini di età compresa tra 8 e 9 anni. Le tre fasi sono state implementate in 3 giorni, uno per ogni fase. Ogni incontro è durato circa 3 ore e si è svolto nell'orario scolastico.

Le attività svolte sono quelle descritte nel par. 4.2. In particolare, la Fase 3, in questo caso, ha riguardato La caccia al tesoro. Le attività si sono svolte, come progettato per tutto il dispositivo, in un'alternanza di lavoro in gruppo e discussioni collettive, con la presenza del docente di matematica o di quello di italiano e di 3 ricercatori (tre degli autori di questo lavoro). II ruolo degli insegnanti è stato di coordinatori, mentre i ricercatori hanno svolto il ruolo di scaffolders (Wood et al., 1976), concretizzatosi nel dare input iniziali per lo svolgimento delle consegne, per poi lasciare che i bambini 
interagissero tra loro per giungere alla soluzione del compito proposto, fungendo da guida durante le attività e, in particolare, nelle discussioni collettive finali.

Durante l'implementazione del dispositivo educativo è stata effettuata una prima osservazione che ha portato a raccogliere dati tramite note osservative e tracce osservabili, riguardante i processi di interazione e negoziazione, che ha permesso di rilevare come tutti i bambini siano stati coinvolti nello svolgimento delle diverse attività didattiche, anche se con un diverso livello di partecipazione. L'osservazione delle attività ci ha portato, inoltre, a focalizzare l'attenzione su vari aspetti da analizzare successivamente, come, ad esempio, la procedura di costruzione del linguaggio da parte dei bambini, la modalità di manipolazione del linguaggio costruito e la gestione delle interpretazioni attribuite al linguaggio creato.

In un secondo momento è stata effettuata l'analisi qualitativa degli altri dati collezionati (video-registrazioni, audio-registrazioni delle attività e delle interviste), che ha fatto emergere in che modo i bambini hanno creato scenari di riferimento "spontanei", risultanti dalla libera discussione tra i partecipanti durante le attività. Nello studio che presentiamo i bambini sono stati intervistati in itinere, dopo le prime due fasi, e alla fine del percorso.

I risultati che discutiamo derivano sia dall'osservazione delle attività che dall'analisi del contenuto effettuata sulle trascrizioni delle interviste (Berelson, 1952; Blanchet, 1985). Questo tipo di analisi ha fatto emergere il modo di elaborare e ridefinire le attività da parte dei bambini. L'analisi è stata condotta su tre livelli (Doise, 1986; Doise et al., 1992):

1. analisi degli aspetti interindividuali: le modalità di apprendimento, le rappresentazioni delle attività e dei compiti derivanti dal lavoro in gruppo;

2. analisi degli aspetti intraindividuali: le procedure attraverso cui i bambini, individualmente, elaborano a livello cognitivo le attività;

3. analisi delle relazioni tra gli aspetti interindividuali e intraindividuali: se e come gli aspetti interindividuali e quelli intraindividuali sono legati tra loro.

In particolare, relativamente a questi tre livelli, sono emersi quattro temi principali, che indichiamo con TP1, TP2, TP3, TP4 (Tabella 1).

\begin{tabular}{|l|}
\hline Temi principali emersi dall'analisi delle interviste \\
\hline TP1 Mancanza di accordo comunicativo \\
\hline TP2 Elaborazione sociale ed individuale delle attività \\
\hline TP3 Produzione dei simboli \\
\hline TP4 Trasformazione del pensiero individuale \\
\hline
\end{tabular}

Tabella 1. Temi principali.

Il primo tema principale, TP1 Mancanza di accordo comunicativo, rientra nel livello interindividuale; i successivi due, TP2 Elaborazione sociale e individuale delle attività e TP3 Produzione dei simboli, appartengono al terzo livello di analisi, poiché riguardano sia il livello interindividuale che quello intraindividuale; infine, nel livello intraindividuale ritroviamo il TP4 Trasformazione del pensiero individuale, come conseguenza di una attività sociale.

\subsection{Mancanza di accordo comunicativo}

A un livello interindividuale sembra che la cooperazione e la competizione (i bambini vengono divisi 
in gruppi che sono in competizione tra loro) giochino un ruolo importante. Questo tipo di attività permette al bambino di riconoscere e comprendere l'esistenza di altri punti di vista e di soluzioni differenti dello stesso problema, senza imposizioni di regole dall'esterno (Carugati \& Selleri, 2005). Inoltre, la creazione condivisa di un linguaggio e la sua manipolazione permettono di acquisire la consapevolezza che i simboli dipendono dal contesto d'uso e che presuppongono un accordo nella comunità dei ricevitori (Ferrari, 2002).

Dall'analisi è emerso che in alcuni momenti critici delle attività ci sono stati dei fallimenti che sembrano siano dovuti proprio ad una mancanza di accordo comunicativo. Questo emerge in particolare dalle interviste riguardanti le attività della Fase 3. Durante lo svolgimento della caccia al tesoro c'è stato un momento in cui un bambino doveva dettare a un altro una sequenza di simboli del linguaggio costruito e manipolato nelle fasi precedenti. II bambino che riceveva la sequenza doveva usarla per raggiungere un obiettivo (trovare il tesoro), ma non ci è riuscito. Da sottolineare che in questa fase non erano state date istruzioni esplicite su se e come raggiungere un accordo. Riportiamo di seguito alcuni stralci delle interviste ${ }^{6}$ riguardanti questo tema principale.

Prot. 2 - IV primaria, F (mancanza di accordo comunicativo)

21. I: «E secondo te come mai non era in grado di dare i comandi?»

22. P: «Perché (.) Perché (.) non sono arrivati a un accordo su nord sud e (.) sulla posizione da cui dovevano partire».

Prot. 4 - IV primaria, M (mancanza di accordo comunicativo)

14. P: «(.) eravamo girati di spalle io e M. e le ho detto alcune cose (.) ma lei non sapeva dove fosse il nord sud est ovest».

Nel primo stralcio c'è la testimonianza di una bambina che ha osservato dall'esterno lo svolgimento della comunicazione della sequenza, che sottolinea un mancato accordo su due fattori importanti: la posizione iniziale di partenza e la posizione dei punti cardinali. Nel secondo stralcio, invece, il bambino intervistato è quello che ha dovuto dettare alla sua compagna di classe M. la sequenza dei simboli. L'intervistato evidenzia come M. non si sia accordata con lui su come posizionare i punti cardinali sul foglio. In entrambi i casi emerge che i bambini sono consapevoli del fallimento riguardante questo episodio e nel descriverlo lo hanno attribuito proprio a un non accordo nella comunicazione - in particolare sulla posizione dei punti cardinali - tra i due bambini impegnati nella caccia al tesoro (colui che comunica e colui che riceve la sequenza di simboli).

Da sottolineare che questi primi risultati ci hanno spinto a progettare un dispositivo educativo che includesse ulteriori attività, il cui obiettivo fosse supportare nei bambini lo sviluppo di una maggiore consapevolezza sulla non unicità dell'interpretazione e sull'importanza di accordi comunicativi all'interno di una comunità che utilizza quel linguaggio (Coppola et al., 2015, 2019).

\subsection{Elaborazione sociale e individuale delle attività}

Unendo i livelli di analisi interindividuale e intraindividuale, è emerso un altro tema principale, che chiamiamo elaborazione sociale e individuale delle attività. Grazie all'analisi effettuata è stato possibile osservare un cambiamento nel pensiero individuale dei bambini, essenzialmente basato sull'attivazione di un processo di rielaborazione - prima sociale e poi personale - dei concetti coinvolti, e su una rappresentazione dell'importanza della collaborazione/cooperazione nello svolgimento dei

6. Le interviste sono state trascritte integralmente utilizzando una versione alleggerita del sistema jeffersoniano. Sono stati utilizzati solo alcuni codici di trascrizione quali: (.) per indicare una micro pausa; (0.5) per indicare la durata di una pausa espressa in decimi di secondo (Jefferson, 1985) 
compiti. I bambini hanno elaborato a livello intraindividuale i compiti, cercando di superare i problemi attraverso la collaborazione con gli altri.

Nel progettare e implementare il dispositivo educativo ci aspettavamo che i bambini aderissero a un modello condiviso e che creassero un linguaggio attraverso una negoziazione. In tal modo volevamo provocare una riflessione dei bambini sui diversi modi di rappresentare un movimento del robot (linguaggio quotidiano, simboli, rappresentazione grafica) e, viceversa, su come interpretare i simboli di un linguaggio. Dall'analisi è emerso che i bambini hanno elaborato le attività e hanno utilizzato diverse rappresentazioni per eseguire il compito e giungere ad una soluzione della situazione problematica.

Prot. 2 - IV primaria, $\mathrm{F}$ (elaborazione sociale e individuale delle attività)

37. I: «Riprendendo il percorso ti ricordi che ad un certo momento ti chiediamo di scrivere e disegnare il percorso su un foglio (.) hai notato qualche differenza tra il momento in cui dovevi disegnare il percorso su un foglio ed il momento in cui dovevi descriverlo parlando?»

38. P: «Era più facile disegnarlo (.) sì (.)».

39. I: «Come mai dici che era più facile disegnarlo?»

40. P: «Perché vedi com'è il percorso (.) com'è e poi lo disegni sul terreno (.) cioè (.) è come se lo disegnassi e poi vedi se il robot può arrivare al punto».

Prot. 1 - IV primaria, M (elaborazione sociale e individuale delle attività)

31. I: «Ti ricordi la penultima volta che ci siamo incontrati ti abbiamo detto di disegnare le istruzioni su un foglio (0.5) era più facile o più difficile dirle a voce o disegnarle?»

32. P: «Era più facile disegnarle su un foglio perché dopo (.) puoi anche dimenticarle (.)».

In entrambi gli stralci di intervista i bambini affermano che è più facile disegnare le istruzioni sul loro foglio che raccontarle a voce. II primo bambino ha bisogno di scrivere perché il disegno lo aiuta a "vedere" il percorso. II secondo dice che il disegno alleggerisce il suo lavoro nel ricordare le istruzioni. Ciò che risulta evidente da questi stralci è il bisogno dei bambini di ricorrere ancora a una rappresentazione grafica (il disegno) per manipolare i simboli del linguaggio costruito. II disegno, che simula il percorso del robot, li aiuta a "vedere se il robot può arrivare al punto" e quindi a ricordare il significato - legato al contesto delle attività - dei simboli del linguaggio creato. I bambini passano dalla sintassi alla semantica del linguaggio - cioè dai simboli al loro significato (il movimento del robot) appoggiandosi ad un altro modo di rappresentare lo stesso significato (la rappresentazione grafica).

\subsection{Produzione di simboli}

Dalla nostra osservazione sembra che sia la negoziazione dei significati dei simboli del linguaggio creato sia la condivisione degli scopi delle attività, abbiano favorito la comprensione della funzione svolta dai simboli - in relazione a quel contesto - e supportato la loro manipolazione. In accordo con la prospettiva vygotskiana, il linguaggio è stato costruito come risposta a vincoli comunicativi e rappresentativi (Ferrari, 2004; Halliday, 1985) e non in conformità a pattern prestabiliti. Le attività proposte avevano come scopo anche quello di supportare nei bambini una maggiore flessibilità nell'uso dei linguaggi. Secondo Ferrari (2002, p. 354) pensiamo che questo tipo di attività «forzano un uso sempre più raffinato della lingua come risposta a specifiche esigenze e vincoli posti esplicitamente dal contesto». Dall'osservazione emerge come il linguaggio finale sia stato costruito dai bambini in modo abbastanza spontaneo, attraverso un passaggio graduale dal linguaggio quotidiano ad un sistema simbolico. Questo cambiamento è stato stimolato attraverso compiti progettati ad hoc. Nella prima fase del dispositivo educativo, così come descritto nel par. 4.2, è stato chiesto a tutti i gruppi di bambini di descrivere con alcune istruzioni i passi del "bambino-robot" in movimento nella stanza. 
In un primo momento i bambini hanno usato il linguaggio quotidiano per descrivere i movimenti del robot, usando espressioni come fare un passo avanti, girare a destra, fare un passo a sinistra e così via. Dopo un po' hanno trovato il linguaggio usato scomodo per il loro scopo, cioè far muovere il robot e raggiungere la soluzione al problema velocemente. Questo momento ha prodotto nei bambini un bisogno di sintesi. Così hanno deciso di usare un simbolo per ogni istruzione, per esempio: $A$ per indicare l'istruzione fare un passo avanti, I per indicare fare un passo indietro e così via. I ricercatori non hanno dato suggerimenti sulla scelta dei simboli. II TP3 produzione di simboli è collegato proprio a queste osservazioni.

Prot.1 - IV primaria, M (produzione di simboli)

23. I: «Quando l'istruzione da più lunga è diventata più breve, giocare era più difficile o più facile?»

24. P: «Con l'istruzione più breve era più facile (.)».

25. I: «Come mai?»

26. P: «Perché dobbiamo scrivere più piccolo (.) ed era più facile da capire (.) ed era possibile risparmiare spazio».

In quest'ultimo stralcio appare come i bambini abbiano trovato più facile gestire il linguaggio simbolico invece del linguaggio quotidiano per risolvere la situazione problematica. Usando i simboli, riferendosi alle attività della Fase 2, sono riusciti anche a semplificare alcune parole (sequenze) della nuova lingua da loro creata. Durante lo svolgimento delle attività i simboli manipolati sono stati intesi dai bambini come "strumenti" per supportare l'esecuzione dei compiti nel contesto in cui stavano agendo (Radford, 2000), come è possibile osservare nello stralcio seguente:

Prot.2 - IV primaria, F (produzione di simboli)

23. I: "Ti ricordi che i comandi erano lunghi? Poi abbiamo giocato un po' con loro e li abbiamo fatti diventare più piccoli (.) Cosa ne pensi? Quando sono diventati più piccoli (.) era più facile o più difficile?»

24. P: «Sì, è diventato più facile perché siamo stati in grado di semplificarli».

25. I: «Così con la semplificazione il gioco è diventato più facile».

26. P: «Sì (.) sì».

\subsection{Trasformazione del pensiero individuale}

II TP4 trasformazione del pensiero individuale riguarda il modo in cui i bambini hanno elaborato e interiorizzato le attività. A un livello di analisi intraindividuale sembra che ci sia stata una trasformazione del pensiero individuale dei bambini (superamento del pensiero egocentrico) come conseguenza delle attività di manipolazione sociale e linguistica (livello interindividuale). I bambini sono diventati consapevoli dell'esistenza di punti di vista diversi e di opinioni diverse dalle loro.

Nelle interviste i bambini hanno dichiarato di aver vissuto un "momento di crisi", quando, in una delle attività della Fase 1, è stato introdotto un secondo robot. In questa attività i due robot erano collocati in posizioni speculari. Ai gruppi è stato chiesto di dare istruzioni ai due robot per far sì che raggiungessero simultaneamente la stessa posizione fissata. Questo compito è stato progettato con I'obiettivo di stimolare uno slittamento dal punto di vista dell'unico robot inizialmente coinvolto a quello dei due robot ora in movimento. La crisi era dovuta al fatto che i bambini non erano in grado di far raggiungere ai due robot la stessa posizione fissata, simultaneamente. Questa nuova situazione problematica ha fatto nascere nei bambini la necessità di rendere il linguaggio più oggettivo e indipendente dal robot, eliminando i riferimenti troppo espliciti al bambino che si muoveva. In tal modo si è passati ad un linguaggio simbolico con un livello maggiore di astrazione dal contesto. I bambini 
hanno anche dichiarato che, grazie al lavoro di squadra e alla cooperazione, hanno superato il momento critico attraverso I'introduzione dei punti cardinali Nord, Sud, Est e Ovest (riferimenti esterni), al posto dei riferimenti interni (come sinistra, destra) relativi a un singolo bambino e legati al contesto.

Prot.1 - IV primaria, M (trasformazione del pensiero individuale)

13. I: «Ad un certo punto se ti ricordi (.) i robot sono diventati due e c'è stato un momento di crisi tra i tuoi compagni (.) secondo te come mai?».

14. P: «Perché io ero qui (.) e l'altro robot era lì (0.5) e uno stava a destra e l'altro a sinistra e per arrivare io e Y. abbiamo fatto dei passi uguali (.) ma ci trovavamo sempre in posizioni diverse (.) quindi abbiamo inventato nord sud est e ovest».

Nello stralcio precedente (intervento 14), il bambino, che era uno dei due robot, ha usato l'espressione "abbiamo inventato il nord, il sud, l'est e l'ovest" come se nord, sud, est e ovest non fossero oggetti già esistenti da poter usare anche in quel contesto, ma nuove istruzioni costruite da loro per superare il loro problema.

Di seguito c'è un altro estratto riguardante questo "momento di crisi" e la modalità di superamento messa in atto dai bambini:

Prot.2 - IV primaria, F (trasformazione del pensiero individuale)

9. I: «Ripensa a questo momento (.) quando ha dato gli ordini (.) inizialmente c'era solo un robot che era R. (.) poi a un certo punto si è deciso di introdurre un altro robot e ce n'erano due (.) in quel momento (.) è cambiato qualcosa?».

10. P: «Sì».

11. I: «Cosa?»

12. $\mathbf{P}$ : «Perché erano in due posizioni diverse (.) quindi non si poteva farli arrivare bene (.) entrambi (.) allo stesso punto».

13. I: «E quando hai dato gli ordini quali difficoltà ha osservato?».

14. P: «(.) perché (.) uno si girava da un lato e un altro da un altro (.) quindi (.) non si poteva metterli entrambi sullo stesso lato».

15. I: «E poi abbiamo risolto questo problema?»

16. $\mathrm{P}:$ : Sì».

17. I: «E come l'abbiamo risolto?»

18. P: «Con le istruzioni nord e sud (.) est e ovest (0.5)».

\section{Discussione e conclusioni}

In questo lavoro abbiamo presentato una serie di riflessioni alla luce di diversi studi effettuati in questi anni sul legame tra linguaggio e sviluppo di abilità logiche. In queste riflessioni abbiamo anche voluto sottolineare l'importanza della progettazione del dispositivo educativo, basato su attività linguistico-manipolative. Per noi ricercatori è stata fondamentale l'analisi derivante dall'implementazione di un primo dispositivo educativo progettato per una quarta classe primaria, descritta in questo lavoro, grazie alla quale sono emersi quattro temi principali riguardanti le modalità di rielaborazione delle attività, che risultano dall'intreccio di aspetti individuali, relazionali e contestuali.

Tali risultati hanno costituito una base di partenza per rimodulare e/o modificare alcune attività, sperimentate in altre classi negli anni successivi (Coppola et al., 2011b, 2014, 2015, 2019). In ogni implementazione il design delle attività è sempre stato funzionale all'apprendimento della matema- 
tica. Infatti, il focus delle attività ha riguardato non solo lo sviluppo di una competenza simbolica nei bambini coinvolti, ma anche l'apprendimento di alcune nozioni matematiche particolari - come il concetto di equivalenza (Coppola et al., 2011b, 2014) - in una continua interazione tra sintassi e semantica del linguaggio, che, in accordo con molti studi, è fondamentale per lo sviluppo del pensiero logico matematico. L'analisi qualitativa ed interpretativa dei dati raccolti si è focalizzata prevalentemente su come i bambini hanno elaborato le attività linguistico-manipolative, in cui erano immersi. Da essa sembra emergere come le attività progettate - e la modalità con cui sono state organizzate - abbiano stimolato interazioni sociali, discussioni e negoziazioni. I bambini sono stati liberi di interpretare le attività e i compiti e di prendere, di conseguenza, iniziative: sono riusciti, in poco tempo, a scegliere, di volta in volta, la strategia adatta per risolvere una situazione problematica. In particolare, hanno negoziato i significati da attribuire al linguaggio simbolico creato, in base al contesto d'uso (Ferrari, 2002), diventando più abili nella manipolazione del linguaggio stesso, che a sua volta è diventato uno strumento per portare a compimento le azioni richieste dal contesto e dalle attività in cui erano impegnati (Radford, 2000). Sembra che nelle attività ci sia stato un cambiamento nel ruolo del linguaggio che da strumento di comunicazione è diventato un oggetto da manipolare per $\mathrm{i}$ bambini per risolvere i compiti loro assegnati.

Dall'analisi delle interviste sembra che ci sia stato quello che Vygotskij (1990) definisce il passaggio dall'interpsichico all'intrapsichico: lavorando in gruppo e manipolando collettivamente il linguaggio, in particolare i suoi simboli, assegnandogli nuovi significati, i bambini hanno potuto elaborare prima socialmente il problema, trovando una soluzione che ha ampliato i loro schemi di conoscenza; successivamente, si sono appropriati dei concetti e li hanno rielaborati in modo individuale.

Più in generale, nel corso degli anni, abbiamo, infatti, osservato (ad esempio, Coppola et al., 2019) che attività basate sulla manipolazione di oggetti linguistici, ambientate in uno spazio dialogico di confronto che favorisca scambi comunicativi (Bakhtin, 1981; Vygotskij, 1990), interazioni e negoziazioni, possono essere in grado di attivare nei bambini processi di riflessione sulla conoscenza matematica. Attraverso le discussioni (alunno-alunno; alunno-insegnante; alunno-ricercatore), infatti, i bambini hanno messo in atto modalità di negoziazione e forme di mediazione che hanno portato a un processo di co-costruzione delle conoscenze (Bruner, 1990; Vygotskij, 1978), trasformando lo spazio in uno spazio per il pensiero (Mollo, 2018; Perret-Clermont, 2001, 2005, 2015). Il linguaggio stesso, creato e manipolato dai bambini, è diventato uno strumento di mediazione che ha permesso di attivare in loro una appropriazione dei concetti in gioco, che potrebbe successivamente aiutarli nel relativo processo di interiorizzazione (Bartolini Bussi \& Mariotti, 2009; Vygotskij, 1990).

L'analisi dei dati ha evidenziato come molti dei bambini che hanno partecipato alle nostre ricerche abbiano mostrato di aver raggiunto un buon livello di consapevolezza riguardo all'uso dei simboli, alla differenza tra i simboli del linguaggio ed il loro significato, le regole per combinarli e manipolarli, e riguardo all'idea che uno stesso linguaggio può avere interpretazioni diverse. La costruzione di sistemi di simboli via via meno legati al contesto, attraverso l'interiorizzazione di punti di vista differenti dai propri ha permesso il raggiungimento di un livello di astrazione del linguaggio più elevato rispetto all'inizio.

Sono stati spesso osservati, durante l'implementazione delle attività, momenti che i bambini stessi hanno riconosciuto come "momenti di crisi". Si pensi, per esempio, al momento in cui si è passati da un solo robot a due robot messi a specchio oppure al momento in cui un compito non è stato portato a termine completamente nell'attività della caccia al tesoro, entrambi descritti in questo lavoro. Nel primo momento di crisi l'esecuzione del compito ha reso i bambini consapevoli del fallimento, dovuto, in questo caso, all'applicazione di schemi cognitivi insufficienti per fornire una risposta corretta. Ma è stata proprio tale situazione che ha determinato un conflitto cognitivo (Piaget, 1923), portandoli ad una trasformazione del pensiero individuale. Da sottolineare che, dalle interviste risulta che solo grazie alla collaborazione con gli altri è stato possibile superare il momento di "impasse" ed a far nascere nei bambini il bisogno di produrre simboli legati ad un linguaggio più oggettivo 
e indipendente dal contesto. Nel secondo caso, invece, sebbene il momento di crisi non sia stato superato durante lo svolgimento dell'attività, emerge dall'analisi delle interviste di esplicitazione il raggiungimento di una certa consapevolezza da parte dei bambini del loro fallimento, interpretato come una mancanza di accordo comunicativo tra i membri del gruppo. Questo ci porta ad affermare come le interviste realizzate abbiano rappresentato uno strumento utile non solo per noi ricercatori ma anche per i bambini, permettendo di ricostruire tutti i processi messi in atto durante la risoluzione delle situazioni problematiche. In tal senso le interviste potrebbero rappresentare un utile strumento didattico anche per gli educatori e gli insegnanti per poter comprendere la natura e la causa di un fallimento o di un successo dei propri studenti.

Per quanto riguarda il design del dispositivo educativo ci sono aspetti che potrebbero essere modificati e migliorati. Ad esempio, in accordo con le ricerche che evidenziano come possa essere importante lo storytelling nell'insegnamento e apprendimento della matematica (Zazkis \& Liljedahl, 2009), sarebbe interessante arricchire e rendere più consistente la cornice narrativa, in modo da ottenere un background per l'intero percorso che possa supportare i processi di apprendimento.

D'altra parte, riteniamo che il dispositivo educativo, insieme alle riflessioni sorte in seguito all'osservazione della sua implementazione - descritte in questo lavoro - possano rappresentare uno spunto per docenti ed educatori che vogliano svolgere attività di manipolazione del linguaggio. Si tratta, ovviamente, di studi qualitativi e situati che, dunque, in un contesto diverso potrebbero fornire risultati diversi. Tuttavia, le attività descritte, e i relativi risultati, potrebbero fornire indicazioni agli insegnanti per stimolare i bambini ad inventare un sistema di segni, che risponda a scopi condivisi, per risolvere problemi.

«L'introduzione delle notazioni matematiche come prodotto esistente non è quindi la migliore occasione di costruire i legami tra testi e scopi, perché questi ultimi sono troppo lontani o sfuggenti. La capacità di mettere insieme tali legami deve essere quindi costruita prima, senza spaventarsi se gli studenti costruiscono notazioni diverse da quelle standard».

(Ferrari, 2021, p. 161)

\section{Ringraziamenti}

Desideriamo ringraziare il Prof. Giangiacomo Gerla per i preziosi spunti e le interessanti discussioni che hanno accompagnato la nascita di queste ricerche.

\section{Bibliografia}

Arzarello, F., \& Robutti, O. (2009). Embodiment e multimodalità dell'apprendimento della matematica. L'insegnamento della matematica e delle scienze integrate, 32A-B(3), 243-268.

Bakhtin, M. M. (1981). The dialogical imagination: four essays by M.M. Bakhtin. University of Texas Press.

Bartolini Bussi, M. G., \& Mariotti, M. A. (2009). Mediazione semiotica nella didattica della matematica: artefatti e segni nella tradizione di Vygotskij. L'Insegnamento della matematica e delle scienze integrate, 32A-B, 270294.

Berelson, B. (1952). Content Analysis in Communication Research. Free Press.

Blanchet, A. (1985). L'entretien dans les sciences socials: l'écoute la parole et le sens. Bordas.

Bruner, J. S. (1977). Early social interaction and language acquisition. In H. R. Schaffer (Ed.), Studies in Mother-infant interaction (pp. 271-289). Accademy Press. 
Bruner, J. S. (1990). Acts of meaning. Harvard University Press.

Bruner, J. S. (2002). La fabbrica delle storie. Diritto, letteratura, vita. Laterza.

Carugati, F., \& Selleri, P. (2005). Psicologia dell'educazione. II Mulino.

Coppola, C., Mollo, M., \& Pacelli, T. (2010). Deduzione come manipolazione linguistica: un'esperienza in una scuola primaria. L'educazione matematica, Anno XXXI, serie 1, 2(3), 5-22.

Coppola, C., Mollo, M., \& Pacelli, T. (2011a). An experience of social rising of logical tools: the role of language. International Journal for Mathematics Teaching and Learning. https://www.cimt.org.uk/journal/coppola.pdf

Coppola, C., Mollo, M., \& Pacelli, T. (2011b). The concept of equivalence in a socially constructed language in a primary school class. In M. Pytlak, T. Roland \& E. Swoboda (Eds.), Proceedings of CERME 7 (pp. 1302-1312). University of Rzeszów, Poland.

Coppola, C., Mollo, M., \& Pacelli, T. (2014). Manipolazione di un linguaggio socialmente costruito in una classe di scuola primaria: costruzione del concetto di equivalenza. L'insegnamento della matematica e delle scienze integrate, 37A (1), 7-33.

Coppola, C., Mollo, M., \& Pacelli, T. (2015). The development of logical tools through socially constructed and culturally based activities. In G. Marsico, M. V. Dazzani, M. Ristum \& A. C. Bastos (Eds.), Educational contexts and borders through a cultural lens - Looking inside. Viewing outside. Cultural Psychology of Education, 1 (pp. 163-176). Springer.

Coppola, C., Mollo, M., \& Pacelli, T. (2019). The worlds' game: collective language manipulation as a space to develop logical abilities in a primary school classroom. European Journal of Psychology of Education, 34(4), 783-799. Springer Netherlands.

D’Amore, B. (1999). Scolarizzazione del sapere e delle relazioni: effetti sull'apprendimento della matematica. L'insegnamento della matematica e delle scienze integrate, 22A(3), 247-276.

Doise, W. (1986). Levels of explanation in social psychology. Cambridge University Press.

Doise, W., Clémence, A., \& Lorenzi Cioldi, F. (1992). Représentations sociales et analyses de données. Presses Universitaires de Grenoble.

Doise, W., \& Mugny, G. (1981). Le développment social de l'intelligence. Interéditions.

Doise, W., Mugny, G., \& Perret-Clermont, A. N. (1975). Social interaction and development of cognitive operations. European Journal of Social Psychology, 5, 367-383.

Ferrari, P. L. (2002). Developing language through communication and conversion of semiotic systems. In A. D. Cockburn \& E. Nardi (Eds.), Proceedings of the 26th Conference of the International Group for the Psychology of Mathematics Education vol.2 (pp. 353-360).

Ferrari, P. L. (2003). Abstraction in Mathematics. In L. Saitta (Ed.), The abstraction paths: from experience to concept Vol.358, N.1435 (pp. 1225-1230). Phil.Trans.R.Soc.Lond. B.

Ferrari, P. L. (2004). Matematica e linguaggio. Quadro teorico per la didattica. Pitagora.

Ferrari, P. L. (2021). Educazione matematica, lingua, linguaggi. Costruire, condividere e comunicare matematica in classe. UTET Università.

Ferrari, P. L., \& Gerla, G. (2015). Logica e Didattica della Matematica. La ricerca logica italiana. Collana Centro De Giorgi, Edizioni della Normale.

Gerla, G. (1988). L'insegnamento della logica nelle scuole elementari e medie. La logica matematica nella didattica, Atti del XII Incontro AILA. 
Gerla, G., Sestito, L., \& Vescia, S. (1990). Linguaggi algebrico-procedurali nella scuola elementare: un progetto di ricerca. La matematica e la sua didattica, 4, 39-48.

Halliday, M. A. K. (1985). An introduction to functional grammar. Arnold.

lannaccone, A. (1984). Il conflitto socio-cognitivo. Età Evolutiva, 19, 119-124.

Iannaccone, A. (2010). Le condizioni sociali del pensiero. Unicopli.

lannaccone, A., \& Perret-Clermont, A. N. (1993). Qu'est-ce que s'apprend? Qu'est-ce que se développe? In J. Wassmann \& P. R. Dasen (Eds.), Everyday Cognition (pp. 235-260). Freiburg Universitätsverlag.

Iannaccone, A., \& Ligorio, B. (2001). La Situated Cognition in Italia. Revue Suisse de Psychologie de l'éducation, 439-452.

lannaccone, A., \& Zittoun, T. (2014). Overview: the activity of thinking on social spaces. In T. Zittoun \& A. lannaccone (Eds.), Activities of thinking in social spaces (pp. 1-12). Nova Science Publishers.

Jefferson, G. (1985). An Exercise in the Transcription and Analysis of Laughter. In T. Van Dijk (Ed.). Handbook of Discourse Analysis, 3 (pp. 25-34). Academic Press.

Kieran, C. (1992). The learning and teaching of school algebra. In D. Grouws (Ed.), Handbook of research on mathematics teaching and learning (pp. 390-419). Simon \& Schuster.

Leont'ev, A. N. (1978). Activity, consciousness, and personality. Prentice-Hall. (Titolo originale: Dejatel'nost, Soznanie, Lichnosf pubblicato nel 1975).

Ligorio, M. B., \& Pontecorvo, C. (2010). La scuola come contesto. Carocci.

Ministero dell'istruzione, dell'università e della ricerca. (2012). Indicazioni nazionali per il curricolo della scuola dell'infanzia e del primo ciclo d'istruzione. http://www.indicazioninazionali.it/2018/08/26/indicazioni-2012/

MacGregor, M., \& Stacey, K. (1997). Students' understanding of algebraic notation. Educational Studies in Mathematics, 33(1), 1-19.

Mollo, M. (2018). II potere intrinseco dell'argomentazione nei processi di apprendimento. In P. Maturi, F. Piro, L. M. Sicca, M. Squillante \& M. Striano (A cura di), Sfide didattiche. Il pensiero critico nella scuola e nell'università (pp. 121-142). Editoriale Scientifica.

Papert, S. (1980). Mindstorms: Children, Computers, and Powerful Ideas. Basic Books.

Piaget, J. (1923). Le langage et la pensée chez l'enfant. Delachaux et Niestlé.

Perret-Clermont, A. N. (1979). La construction de l'intelligence dans l'interaction sociale. Peter Lang, coll. Exploration.

Perret-Clermont, A. N. (2001). Psychologie sociale de la construction de l'espace de pensée. In J. J. Ducret (Ed.), Actes du Colloque «Constructivismes: usages et perspectives en éducation», 8, (pp. 65-82). Service de la recherche en éducation.

Perret-Clermont, A. N. (2004). The thinking spaces of the young. In A. N. Perret-Clermont, C. Pontecorvo, L. Resnick, T. Zittoun \& B. Burge (Eds.), Joining society: social interactions and learning in adolescence and youth (pp. 3-10). Cambridge University Press.

Perret-Clermont, A. N. (2005). Lo spazio per il pensare. In C. Pontecorvo (A cura di), Discorso e apprendimento. Una proposta per l'autoformazione degli insegnanti (pp. 21-34). Carocci Editore. 
Perret-Clermont, A. N. (2015). The architecture of social relationships and thinking spaces for growth. In C. Psaltis, A. Gillespie \& A. N. Perret-Clermont (Eds.), Social Relations in Human and Societal Development (pp. 51-70). Palgrave Macmillan.

Perrin-Glorian M. J. (1994). Théorie des situations didactiques: naissance, développement, perspectives. In M. Artigue, R. Gras, C. Laborde \& P. Tavignot (Eds.), Vingt ans de didactique des mathématiques en France. Hommage à Guy Brousseau et Gérard Vergnaud (pp. 97-148). La Pensée Sauvage.

Planas, N. (2018). Language as resource: a key notion for understanding the complexity of mathematics learning. Educational Studies in Mathematics, 98, 215-229.

Planas, N., Morgan, C., \& Schütte, M. (2018). Mathematics and language: lessons and directions from two decades of research. ERME Handbook. Routledge.

Radford, L. (2000). Signs and meanings in students' emergent algebraic thinking: a semiotic Analysis. Educational Studies in Mathematics, 42, 237-268.

Radford, L., Bardini, C., Sabena, C., Diallo, P., \& Simbagoye, A. (2005). On embodiment, artifacts, and signs: a semiotic cultural perspective of mathematical thinking. In H. L. Chick \& J. L. Vincent (Eds.), Proc. of the 29th Conf. of the Intern. Group for the Psychology of Math. Education, 4 (pp. 113-120). PME.

Sfard, A. (2001). There is more to discourse than meets the ears: looking at thinking as communicating to learn more about mathematical learning. Educational Studies in Mathematics, 46, 13-57.

Vermersch, P. (2005). Descrivere il lavoro. Carocci Editore. (Titolo originale: L'entretien d'explicitation pubblicato nel 1994).

Vygotskij, L. S. (1978). Mind in Society: The development of higher psychological processes. Harvard University Press.

Vygotskij, L. S. (1990). Pensiero e Linguaggio. Laterza.

Vygotskij, L. S. (1997). The history of the development of higher mental functions. In R. W. Rieber \& A. S. Carton (Eds.), The collected works of L.S. Vygotskij Vol. 4. Plenum Press.

Wartofsky, M. (1979). Models. D. Reidel.

Wood, D. J., Bruner, J. S., \& Ross, G. (1976). The role of tutoring in problem solving. Journal of Child Psychiatry and Psychology, 17(2), 89-100.

Zazkis, R., \& Liljedahl, P. (2009). Teaching mathematics as storytelling. Sense Publishers.

Zucchermaglio, C. (1996). Vygotskij in azienda. Apprendimento e comunicazione nei contesti lavorativi. Carocci. 